Ciência Florestal, Santa Maria, v. 22, n. 2, p. 295-303, abr.-jun., 2012

ISSN 0103-9954

\title{
CALIDAD DEL CARBONO ORGÁNICO DEL SUELO EN DIFERENTES TÉCNICAS DE MANEJO DE RESIDUOS FORESTALES
}

\author{
SOIL ORGANIC CARBON QUALITY IN DIFFERENT FOREST RESIDUE MANAGEMENT
}

Ana Maria Lupi ${ }^{1}$ Marta E. Conti ${ }^{2}$ Roberto A. Fernandez ${ }^{3}$

\section{RESUMEN}

Las distintas técnicas de cosecha y manejo de residuos pueden provocar cambios en la cantidad y calidad de la materia orgánica (MO) del suelo. El objetivo de este trabajo fue evaluar las modificaciones en la concentración y la calidad de la MO en un Ultisol con diferentes manejos de los residuos de la cosecha forestal del Pinus elliottii Engelm, al inicio de la segunda rotación. Los tratamientos analizados fueron: a) extracción manual de los residuos de la cosecha y del piso forestal, suelo descubierto (ER), b) quema de residuos y del mantillo, y laboreo con rastra (PI), c) conservación de residuos y del mantillo sobre el suelo (PC). Se determinó el carbono orgánico (CO) en todo el suelo y en las fracciones de ácidos fúlvicos (AF), ácidos húmicos $(\mathrm{AH})$, huminas $(\mathrm{HU})$ y en la fracción liviana (CL). La conservación de los residuos de la cosecha no aumentó el CO ni el CL. Se observó una pérdida de la fracción de ácidos fúlvicos (AF) particularmente en PI. Los tratamientos sin residuos forestales (ER y PI) muestran un aumento en el grado de estabilización de la MO con un incremento de las HU y de la relación CAH/CAF.

Palabras claves: sustancias húmicas; residuos de cosecha; preparación del terreno; Pinus.

\section{ABSTRACT}

Different harvest techniques and residue management can cause changes in the quantity and quality in soil organic matter $(\mathrm{OM})$. This study aims to assess changes in concentration and in the quality of the $\mathrm{OM}$, in an Ultisol with differents forest residue managements at the beginning of the second rotation. The treatment was : a) elimination of harvest residues and forest floor, bare soil (RE), (b) burning of harvest residues and forest floor and subsequent plowing (IP), (c) maintenance of harvest residues and forest floor on the ground (CP). Soil samples were analyzed for determination of soil organic carbon (OC), carbon in the light fraction (LC) and carbon in the fulvic acids (FA), humic acids (HA) and humins (HU) fractions. The harvest residue conservation did not increase the OC or the LC. There was a loss of the more labile fraction (FA) particularly in IP. The treatments without forest residues and forest floor (RE and IP) show an increase in the degree of stabilization of the OM with an increase HU and in the ratio HA/FA.

Keywords: humic substances; harvest residues; site preparation; Pinus.

\section{INTRODUCCIÓN}

El manejo de la materia orgánica (MO) en el período inter-rotación es un aspecto crítico para asegurar la productividad de las plantaciones de rápido crecimiento en los ciclos sucesivos (MENDHAM et al., 2003). La aplicación de técnicas intensivas de cosecha y de preparación del sitio pueden provocar un impacto negativo sobre el fnivel y la calidad de la MO (NAMBIAR, 1995). La cosecha forestal altera el ciclo del carbono (C) provocando pérdida de las sustancias húmicas del mantillo y del horizonte superficial del suelo mineral (USSIRI y JHONSON, 2007). Estas alteraciones

1. Ingeniera Forestal, Msc., Investigador del Instituto de Suelos, CIRN - INTA Castelar Los Reseros y Las Cabañas s/n CC25, Villa Udaondo, Castelar, Buenos Aires, Argentina. amlupi@cnia.inta.gov.ar

2. Ingeniera Agrónoma, Msc., Profesor e Investigador de la Cátedra de Edafología, Facultad de Agronomía UBA, Av. San Martín 4453 (1417), Buenos Aires, Argentina. conti@agro.uba.ar

3. Ingeniero Agrónomo, Msc., Investigador de la Estación Experimental Agropecuaria Montecarlo INTA, Av. El Libertador 2472 (3384), Montecarlo, Misiones, Argentina. Profesor de la Cátedra de Edafología, UNAM, Eldorado, Misiones, Argentina.rfernandez@montecarlo.inta.gov.ar

Recebido para publicação em 23/07/2010 e aceito em 26/04/2011 
van acompañadas de cambios en la disponibilidad de los nutrientes, afectando en ciertas ocasiones el crecimiento del cultivo (NZILA et al., 2002; POWERS et al., 2005).

El fuego puede afectar negativamente las propiedades del suelo aún a temperaturas relativamente bajas (NEARY et al., 1999). En este sentido, Jurgensen et al. (1997); Cade-Menun et al. (2000) y Mendham et al. (2003) detectaron disminuciones importantes en el $\mathrm{CO}$ (carbono orgánico) del suelo por efecto de la quema de residuos. Almendros et al. (1990); Shindo y Urabe (1993) y Fernández et al. (1997), explican que durante la acción del fuego se produce una destrucción selectiva de las sustancias húmicas poco evolucionadas, aumentando las formas orgánicas más estables. De acuerdo a la intensidad de la quema, su efecto puede diluirse con el transcurso del tiempo, en el corto plazo (2-5 años) (NEARY et al. 1999). Sin embargo Cromack et al. (1999) rescatan el efecto negativo del fuego luego de 10 años de su aplicación y señalan que la fracción liviana del C fue la variable más sensible a los cambios ocurridos por el manejo. De una manera similar al efecto del fuego, el laboreo favorece la oxidación de la MO más lábil, aumentando la predominancia de las fracciones más estables (PICCOLO, 1996; BAYER et al., 1999).

Las nuevas técnicas de manejo interrotación como la conservación de los residuos de la cosecha demostró ser una práctica eficiente para mitigar los impactos negativos ocasionados por las técnicas intensivas (NAMBIAR, 1995; GONÇALVEZ et al. 2004); particularmente con efectos más pronunciados en sitios de baja fertilidad natural. Mathers et al. (2003) y Chen y Xu (2006) reportan un aumento en la cantidad y calidad de la MO edáfica, como así también en la disponibilidad de nutrientes esenciales como el $\mathrm{N}$ (nitrógeno) al mantener sobre el suelo los residuos forestales.

La MO del suelo es empleada como indicador del estado o salud del mismo por su contribución a los procesos vitales del suelo dentro del ecosistema (KÖRSCHENS et al. 1998). Se disponen de diferentes métodos de extracción de la MO edáfica. El uso de métodos químicos de fraccionamiento de la MO permite diferenciar las sustancias húmicas de acuerdo a su solubilidad, reactividad, composición molecular entre otras cosas. El ciclaje de estos compuestos húmicos, caracterizados por su peso molecular relativamente elevado, puede tardar desde décadas a siglos imprimiéndole un rol importante en el secuestro de $\mathrm{C}$ en el largo plazo. El fraccionamiento físico de los componentes orgánicos del suelo se basa en el rol de los minerales edáficos dentro de la estabilización y el ciclado de la MO; pudiendo sus resultados relacionarse con la estructura y función de la MO in situ (GALANTINI y SUÑER, 2008). Mediante este método es posible obtener la MO menos transformada o libre, que debido a su mayor sensibilidad permite conocer los cambios que se producen en el sistema de producción además de predecir la disponibilidad de los nutrientes en el corto plazo.

En la provincia de Misiones, Argentina, a mediados de la década del 90 una buena parte de los suelos rojos profundos en forestaciones con Pinus terminaron su primer rotación forestal. El método tradicional de preparación del suelo consistía en la eliminación de los residuos forestales y de la vegetación del sotobosque con quema, seguido de laboreo del suelo. La remoción del suelo, como método de control de malezas, se realizaba periódicamente en la entre línea de plantación hasta el segundo o tercer año, coincidentemente con el cierre de la canopia. Con estas técnicas se favorecía la mineralización de la $\mathrm{MO}$, el suelo permanecía descubierto y expuesto a procesos de erosión. Con el inicio del segundo ciclo, algunas empresas forestales en conjunto con el INTA (Instituto Nacional de Tecnología Agropecuaria) comenzaron a experimentar nuevas alternativas de manejo que consideren la eliminación de la quema de residuos acoplada a un laboreo localizado (subsolado) o la no remoción del suelo.

En suelos Ultisoles y Alfisoles de la región NO (Noroeste) de la provincia de Misiones, la implementación de técnica intensivas de establecimiento (quema más laboreo) produce una reducción en lo niveles de MO total y su fracción liviana, como así también en algunos nutrientes como el N (FERNANDEZ et al., 2000; GIUFFRE et al., 2002). Sin embargo, según el tratamiento que reciben los residuos de la cosecha se puede generar un cambio en las fracciones de la MO, originando de esta manera cambios en la calidad (MATHERS et al., 2003).Los cambios en la calidad de la MO determinan alteraciones en la mineralización y en la disponibilidad de los nutrientes lo cual puede afectar la productividad de las plantaciones.

La hipótesis indica que la conservación de residuos de la cosecha promueve un aumento en la calidad de la MO debido a un incremento en 
la fracción liviana del $\mathrm{CO}$, como así también un incremento en el $\mathrm{CO}$ de las fracciones de baja y media estabilidad. El objetivo de este trabajo fue analizar las concentraciones de $\mathrm{CO}$ en las fracciones separadas por métodos químicos y físicos, en diferentes modalidades de manejo de residuos de la cosecha forestal, a los 29 meses de aplicar los tratamientos.

\section{MATERIALES Y MÉTODOS}

\section{Caracterización del área de estudio}

El estudio se desarrolló en el $\mathrm{NO}$ de la provincia de Misiones, Argentina; ( $25^{\circ} 57^{\prime}$ lat. S y $54^{\circ} 27^{`}$ long. O) (Figura 1). El clima fue clasificado como Cfa en el sistema de Köppen, es subtropical húmedo con precipitaciones que oscilan desde 1900 $-2100 \mathrm{~mm}$ sin mostrar diferencias en su distribución a lo largo del año. La temperatura media anual es de $20^{\circ} \mathrm{C}$ y la amplitud térmica media anual de $11^{\circ} \mathrm{C}$. La vegetación natural original del sitio es la selva subtropical descripta por Cabrera (1976).

El sitio experimental se encuentra ubicado en un ambiente con relieve suave a ondulado (3\% de pendiente), con una altitud aproximada de 200 m snm. El suelo es un Kandiudult (INTA, 1990), con más de 2 metros de profundidad efectiva y bien drenado. Presenta arcillas del tipo de las caolinitas y óxidos de Fe y Al (> $65 \%$ de arcilla en todo el perfil). La secuencia de horizontes característicos de estos suelos es: A, AB, $\mathrm{B}_{\mathrm{t} 1}, \mathrm{~B}_{\mathrm{t} 2}, \mathrm{BC}, \mathrm{C}$. Un muestreo de suelo realizado previo a la aplicación de los tratamientos indica que en la profundidad $0-10 \mathrm{~cm}$ el nivel de CO (WALKEY y BLACK, 1934) fue de $20 \mathrm{~g} \mathrm{~kg}^{-1}$, la concentración de $\mathrm{N}$ total (KJELDAHL) fue de $1,9 \mathrm{~g} \mathrm{~kg}^{-1}$, el $\mathrm{pH}\left(\mathrm{H}_{2} \mathrm{O}\right)$ 5,8, la CIC efectiva fue de $12 \mathrm{cmol}_{\mathrm{c}} \mathrm{kg}^{-1}$ y el $\mathrm{P}$ disponible (BRAY II) menos de 5 ppm.

En el año 1971 se eliminó el bosque nativo y se estableció una plantación de Pinus elliottii Engelm, que en febrero de 1996 alcanzó la edad de corte. Los restos de cosecha fueron distribuidos sobre el terreno y permanecieron durante 6 meses. Se pasó un rolo de $2 \mathrm{Mg}$, con la finalidad de quebrar y aplastar los residuos para favorecer el contacto con el suelo.

\section{Muestreo de residuos de la cosecha}

En julio de 1996, previo a la aplicación de los tratamientos, se realizó un muestreo de residuos en 12 parcelas de $480 \mathrm{~m}^{2}$. Se tomaron 12 muestras de cada unidad de muestreo con un marco de hierro de $0,78 \mathrm{~m}^{2}$ (144 muestras en toda el área del ensayo). Los residuos fueron clasificados en dos clases: (i) finos: mantillo, hojas y ramas con diámetro inferior a $1 \mathrm{~cm}$; y (ii) gruesos: conos, ramas y restos de fustes menores a $15 \mathrm{~cm}$ de diámetro. Luego de registrar el peso húmedo a campo se elaboró una submuestra por parcela de cada clase de residuos y se llevó a estufa $\left(70^{\circ} \mathrm{C}\right)$ hasta peso contante. Se determinó el porcentaje de humedad y se estimó la cantidad de materia seca por hectárea $\left(\mathrm{MS} \cdot \mathrm{ha}^{-1}\right)$. Una muestra de cada clase de material se envió al laboratorio para determinar la concentración de N, P y K.

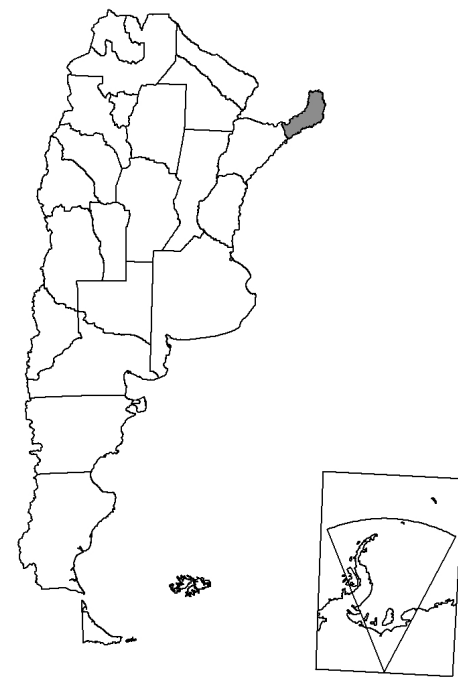

Provincia de Misiones en la República Argentina

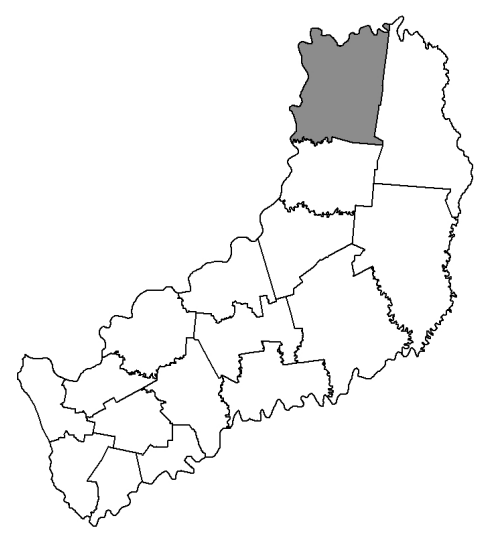

Dapartamento de Iguazú en la Provincia de Misiones

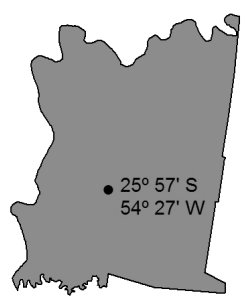

Sitio de estudio en el Departamento de Iguazú

FIGURA 1: Localización del ensayo en la provincia de Misiones, Republica Argentina.

FIGURE 1: Location of the experiment in Misiones province, Argentina. 


\section{Diseño Experimental}

En agosto de 1996 se aplicaron 3 tratamientos bajo un diseño en bloques completos al azar con 4 repeticiones La superficie total de cada unidad experimental (incluyendo una zona buffer) fue de $840 \mathrm{~m}^{2}$, mientras que la superficie neta fue de 480 $\mathrm{m}^{2}$. Los tratamientos aplicados fueron: 1-Extracción manual (ER): Los restos de la cosecha forestal y el mantillo del Pinus elliottii antecesor se extrajeron en su totalidad en forma manual. El suelo permaneció sin cobertura. 2- Preparación intensiva de sitio (PI) o sistema tradicional de la región: se procedió a la quema de los restos de la cosecha y del mantillo y luego se procedió al laboreo con una rastra liviana hasta los $15 \mathrm{~cm}$ de profundidad. Si bien no se cuenta el registro de la temperatura de la quema, en general las que se aplican para la reducción de los restos de la cosecha en bosques implantados son calificadas en el rango de intensidad leve a moderada. Dada la heterogeneidad de la quema, consecuencia de la distribución de los residuos, se elaboró manualmente un croquis identificando dentro de las parcelas las líneas de la plantación anterior y los sectores con cenizas, restos de material parcialmente carbonizado, carbón o suelo con cambios de color producto de la quema. 3- Preparación cero de sitio (PC): los restos de la cosecha fueron mantenidos sobre el suelo sin recibir ningún tipo de laboreo.

En septiembre de 1996 se procedió a la plantación con Pinus taeda L. y el suelo no recibió ningún tipo de fertilizante ni enmienda. El control de malezas se realizó con herbicidas hasta finales del primer año.

\section{Muestreo de suelos y determinaciones de laboratorio}

A los 29 meses de aplicar los tratamientos se tomó una muestra de suelo compuesta por 10-13 piques por parcela, de las profundidades $0-5 \mathrm{~cm}$ y $5-15 \mathrm{~cm}$. El mapeo realizado luego de la quema en el tratamiento PI permitió obtener las muestras de los sectores quemados y laboreados. Las muestras se secaron al aire y fueron pasadas por un tamiz de 0,25 $\mathrm{mm}$ de abertura de malla, tratando de evitar el paso de restos de carbón que pudiera presentar el suelo de las muestras obtenidas del tratamiento PI.

A efectos comparativos se tomaron muestras de suelo en un sitio con vegetación natural (MN) próximo al experimento, bajo similar condición edáfica y topográfica. Se establecieron 4 parcelas de similar superficie a las del experimento y se procedió de similar forma al muestreo. Esta situación no fue incluida en el análisis estadístico ya que no es un tratamiento aleatorizado.

El CL se separó según la metodología propuesta por Richter et al. (1975) y el fraccionamiento químico mediante Richter et al. (1978/79). Mediante Walkley y Black (1934) se cuantificó el CO en el suelo entero, el CO separado en forma física (CL) y el $\mathrm{CO}$ en ácidos húmicos $(\mathrm{CAH})$, en ácidos fúlvicos (CAF) y en huminas (CHU). Los valores de $\mathrm{CO}$ no fueron transformados mediante la utilización de factores.

\section{Análisis estadístico}

Para comprobar el efecto de los tratamientos sobre las variables respuesta se realizó un análisis de varianza bajo un modelo en bloques completos al azar con el paquete estadístico R (DEVELOPMENT CORE TEAM R, 2007). Cuando las diferencias fueron significativas las medias se compararon a través de la prueba de Tukey $(\mathrm{P}<0,05)$. Se analizaron las correlaciones entre las variables.

\section{RESULTADOS Y DISCUSIÓN}

\section{Condición de inicio}

La cantidad de materia seca depositada sobre el suelo luego de la cosecha (hojas, ramas, restos de fuste no comercial y frutos) fue de $40 \mathrm{Mg} \mathrm{ha}^{-1}$ de la cual, el 50\% aproximadamente incluyó el material fino y el $50 \%$ restante, material de características leñosas. No se cuantificó el aporte proveniente de los tocones y raíces remanentes, que constituyen una fuente importante de carbono (C).

Los residuos de la cosecha generan un importante retorno de $\mathrm{C}$ al suelo como sustrato para la descomposición de los microorganismos. Asumiendo que el $50 \%$ de la biomasa aportada es C (IPCC, 2003), la conservación de los restos de cosecha (o la eliminación por extracción y quema) significó un aporte (o pérdida) de $\approx 20 \mathrm{Mg} \mathrm{ha}^{-1}$.

La cantidad de N, P y K contenida en los residuos fue de $297 \mathrm{Kg} \mathrm{ha}^{-1}, 43 \mathrm{Kg} \mathrm{ha}^{-1}$ y 195 $\mathrm{Kg}$ ha $^{-1}$ respectivamente. La aplicación del fuego durante el establecimiento facilita las tareas de plantación y post-plantación, sin embargo puede ocasionar importantes pérdidas de $\mathrm{C}, \mathrm{N}$ y $\mathrm{P}$ por volatilización y trasporte aéreo de cenizas, aun en quemas de intensidad leve y moderada (RAISON, 1979; CARTER y FOSTER, 2004).

\section{Carbono orgánico del suelo (CO)}

La Tabla 1 presenta la concentración de CO 
del suelo en las profundidades $0-5 \mathrm{~cm}$ y $5-15 \mathrm{~cm}$, para cada tratamiento y para una condición de referencia $(\mathrm{MN})$. La concentración de CO varió desde $24 \mathrm{~g}$ $\mathrm{kg}^{-1}$ hasta $27 \mathrm{~g} \mathrm{~kg}^{-1}$ en la profundidad $0-5 \mathrm{~cm} \mathrm{y}$ desde $16 \mathrm{~g} \mathrm{~kg}^{-1}$ y $20 \mathrm{~g} \mathrm{~kg}^{-1}$ en la profundidad 5-15 $\mathrm{cm}$. En ambas profundidades las concentraciones fueron significativamente más bajas en PC. En el ambiente con vegetación natural (MN) los valores de $\mathrm{CO}$ fueron intermedios entre las prácticas intensivas (PI y ER) y la conservacionista (PC).

Contrariamente a lo esperado, la eliminación de los residuos en ER y PI no afectó negativamente los niveles de $\mathrm{CO}$ y puede estar asociado a la baja intensidad de la quema (en PI) y a la estabilización físico-química del $\mathrm{CO}$ con las arcillas y óxidos de $\mathrm{Fe}$ y $\mathrm{Al}$ que ejerce un fuerte efecto protector (SIX et al., 2002). El incremento relativo observado en el $\mathrm{CO}$ del suelo en PI puede ser el resultado de: 1.- la descomposición de las raíces y microorganismos muertos por efecto del calentamiento (FERNASIDE y BARBOSA, 1998); 2.- el ingreso al suelo, por translocación o mezcla, de productos orgánicos originados por la quema del mantillo (McKEE, 1982) y 3.- la presencia de finas partículas de carbón incorporadas a las muestras.

Resulta dificultoso inferiracerca del impacto del fuego en la capa 5-15 cm como consecuencia del laboreo, sin embargo, es esperable que al tratarse de quemas controladas el efecto se limite a unos pocos centímetros superficiales (RAISON, 1979). La muerte de raíces y microorganismos podría proyectarse hasta $\operatorname{los} 5 \mathrm{~cm}$ de profundidad por efecto del calentamiento (DEBANO et al., 1977 citado por NEARY et al., 2000).

La disminución del $\mathrm{CO}$ en PC podría ser producto del conocido fenómeno de naturaleza transitoria llamado "efecto priming" (STEVENSON, 1986; KUZYAKOV et al., 2000). Los residuos de la cosecha presentan compuestos orgánicos con estructuras moleculares complejas que dificultan la biodegradación, por ejemplo: resinas, ligninas, alta relación $\mathrm{C} / \mathrm{N}$ (KÖGELKNABNER,1991). Esto pudo estimular la descomposición del humus nativo del suelo como mecanismo de acceso a fuentes de energía para degradar los residuos.

Nuestros resultados difieren de los obtenidos por Giuffré et al. (2002) en este sitio a los 6 meses de aplicar los tratamientos. Al analizar el CO en la profundidad $0-10 \mathrm{~cm}$, observaron que el tratamiento ER presentó los menores niveles cuando se lo comparó con un tratamiento similar a PC, pero se incluye el laboreo con rastra sobre los residuos.

\section{Carbono Liviano (CL)}

La Tabla 1 presenta la concentración de CL del suelo en las profundidades 0-5 cm y 5-15 $\mathrm{cm}$, para cada tratamiento y una condición de referencia $(\mathrm{MN})$. La conservación o eliminación de los residuos no modificó $(\mathrm{P}>0,05)$ el CL del suelo. Si bien existen factores relacionados con la calidad, tipo y disposición del material que pueden explicar este comportamiento, debemos destacar que los elevados coeficientes de variación de este parámetro (entre el 25\%-40\%) podrían enmascarar la respuesta. Giuffré et al. (2002) reportan diferencias significativas al comparar el CL en la profundidad $0-10 \mathrm{~cm}$ del tratamiento ER versus un tratamiento similar a PC. Los autores también destacan los elevados coeficientes de variación que presenta la variable.

TABLA 1: Carbono orgánico (CO) y carbono liviano (CL) $\left(\mathrm{g} \mathrm{kg}^{-1}\right)$ en las profundidades 0-5 $\mathrm{cm}$ y 5-15 $\mathrm{cm}$, en diferentes sistemas de manejo de residuos forestales y en bosque nativo.

TABLE 1: Soil organic carbon and light carbon in the depths $0-5 \mathrm{~cm}$ and $5-15 \mathrm{~cm}$ in different forest residue managements and native forests.

\begin{tabular}{lllll}
\hline & \multicolumn{3}{c}{ CO } & \multicolumn{2}{c}{ CL } \\
\cline { 2 - 3 } & \multicolumn{1}{c}{$0-5 \mathrm{~cm}$} & $5-15 \mathrm{~cm}$ & $0-5 \mathrm{~cm}$ & \multicolumn{1}{c}{$5-15 \mathrm{~cm}$} \\
PI & $27,81(3,18) \mathrm{a}$ & $19,75(1,61) \mathrm{a}$ & $6,3(1,6) \mathrm{a}$ & $2,8(0,8) \mathrm{a}$ \\
ER & $26,55(2,14) \mathrm{a}$ & $18,28(1,27) \mathrm{a}$ & $5,9(1,7) \mathrm{a}$ & $2,9(1,0) \mathrm{a}$ \\
PC & $24,42(2,55) \mathrm{b}$ & $15,62(1,68) \mathrm{b}$ & $4,9(2,0) \mathrm{a}$ & $2,5(1,7) \mathrm{a}$ \\
MN & $26,15(1,72)$ & $18,49(2,55)$ & $6,5(0,7)$ & $2,4(0,5)$ \\
\hline
\end{tabular}

En que: Letras distintas en cada columna indican diferencias estadísticas a un $\mathrm{P}<0,05$ mediante test de Tukey. Los valores entre paréntesis corresponden al desvío estándar de la muestras. PI: preparación intensiva, ER: extracción de residuos, PC: conservación de residuos, MN: monte nativo. 
El CL se correlacionó en forma significativa con el $\mathrm{CO}$ de la profundidad $0-5 \mathrm{~cm}(\mathrm{r}=0,79 ; \mathrm{P}$ $<0,05)$ y $5-15 \mathrm{~cm}(\mathrm{r}=0,61 ; \mathrm{P}<0,05)$. Teniendo en cuenta que los valores más altos de $\mathrm{CO}$ y $\mathrm{CL}$ se encontraron en el tratamiento PI, es posible que se deba a la presencia de productos orgánicos de baja densidad originados por la quema de los residuos (partículas de carbón o restos parcialmente carbonizados).

En la situación $\mathrm{MN}$ el CL representa aproximadamente el $25 \%$ del CO, valor comparable a lo informado por Duxbury et al. (1989) y Bayer et al. (1999) para suelos regiones tropicales. En las áreas bajo segunda rotación con pinos, las proporciones fueron inferiores a MN lo cual podría sugerir que el desmonte y la puesta bajo cultivo de estos suelos, con técnicas intensivas en primera y segunda rotación, contribuiría a la pérdida de la fracción liviana (GREGORICH y JANZEN, 1996).

La proporción CL/CO en la profundidad $0-5 \mathrm{~cm}$ fue del $22,8 \%$ en PI, $22 \%$ en ER y $20 \%$ en PC. En la profundidad $5-15 \mathrm{~cm}$ los valores mostraron una tendencia opuesta en el orden PC $(16 \%)>$ ER $(15 \%)>$ PI $(14 \%)$. Los valores de CL en subsuperficie fueron 1,7 a 2 veces más bajos a la capa superior, demostrando la estratificación que sufre el CO del suelo, producto del mantillo presente en las plantaciones.

\section{Ácidos Fúlvicos (CAF), Ácidos Húmicos (CAH), Huminas (CHU)}

En la Tabla 2 se encuentran, para cada tratamiento y profundidad de muestreo, las concentraciones medias de $\mathrm{CO}$ en cada extracto obtenido luego del fraccionamiento químico, y su proporción relativa al $\mathrm{CO}$ del suelo entero.

El CAF, conformado por moléculas orgánicas simples, de bajo peso molecular, solubles al agua; representa una pequeña porción de la fracción húmica total (7\%-11\%) y se correlacionó en forma negativa con el $\mathrm{CO}$ del suelo entero $(\mathrm{r}=-0,57$; $\mathrm{P}<0,05)$. Este comportamiento estaría asociado al lavado de compuestos orgánicos solubles de los residuos del tratamiento $\mathrm{PC}$, donde se encontró el valor absoluto y relativo más alto.

La concentración de CAF en la profundidad 0-5 cm fue similar $(\mathrm{P}>0,05)$ para todos los tratamientos, aunque el valor absoluto más bajo se

TABLA 2: Concentración de carbono en ácidos fúlvicos (CAF) en ácidos húmicos (CAH) y en huminas (CHU), y su correspondiente proporción relativa al $\mathrm{CO}$ en diferentes sistemas de manejo de residuos forestales y en bosque nativo.

TABLE 2: Fulvic acid carbon (AFC), húmica acid carbon (HAC) and humins carbon (HUC), and relative proportion to soil organic carbon, in different forest residue managements and native forest.

\begin{tabular}{lcccc}
\hline & \multicolumn{2}{c}{$0-5 \mathrm{~cm}$} & \multicolumn{2}{c}{$5-15 \mathrm{~cm}$} \\
\hline & $\begin{array}{c}\mathrm{CAF} \\
\left(\mathrm{g} \mathrm{kg}^{-1}\right)\end{array}$ & $\begin{array}{c}\mathrm{CAF} / \mathrm{CO} \\
(\%)\end{array}$ & $\begin{array}{c}\mathrm{CAF} \\
\left(\mathrm{g} \mathrm{kg}^{-1}\right)\end{array}$ & $\begin{array}{c}\mathrm{CAF} / \mathrm{CO} \\
(\%)\end{array}$ \\
PI & $2,1(0,4) \mathrm{a}$ & $7,8(1,8) \mathrm{b}$ & $1,3(0,3) \mathrm{c}$ & $6,8(3,5) \mathrm{c}$ \\
ER & $2,4(0,5) \mathrm{a}$ & $9,4(2,3) \mathrm{ab}$ & $2,0(0,4) \mathrm{a}$ & $10,6(5,3) \mathrm{b}$ \\
PC & $2,5(1,0) \mathrm{a}$ & $10,2(3,3) \mathrm{a}$ & $1,9(0,6) \mathrm{b}$ & $12,1(7,4) \mathrm{a}$ \\
MN & $2,8(0,4)$ & $10,7(1,8)$ & $2,0(0,4)$ & $11,1(2,66)$ \\
& $\mathrm{CAH}$ & $\mathrm{CAH} / \mathrm{CO}$ & $\mathrm{CAH}$ & $\mathrm{CAH} / \mathrm{CO}$ \\
\hline PI & $8,0(0,8) \mathrm{b}$ & $29,9(6,2) \mathrm{b}$ & $4,6(0,7) \mathrm{c}$ & $23,6(3,5) \mathrm{b}$ \\
ER & $8,7(0,8) \mathrm{a}$ & $33,8(4,1) \mathrm{a}$ & $7,2(0,7) \mathrm{a}$ & $37,7(5,3) \mathrm{a}$ \\
PC & $7,1(0,9) \mathrm{c}$ & $28,9(2,6) \mathrm{b}$ & $5,51,2) \mathrm{b}$ & $35,0(7,4) \mathrm{b}$ \\
MN & $8,5(1,2)$ & $32,9(3,5)$ & $6,9(0,8)$ & $38,0(6,5)$ \\
& $\mathrm{CHU}$ & $\mathrm{CHU} / \mathrm{CO}$ & $\mathrm{CHU}$ & $\mathrm{CHU} / \mathrm{CO}$ \\
\hline PI & $17,2(3,7) \mathrm{a}$ & $62,1(7,4) \mathrm{a}$ & $13,8(1,4) \mathrm{a}$ & $69,7(3,4) \mathrm{a}$ \\
ER & $15,0(2,5) \mathrm{b}$ & $56,7(5,8) \mathrm{b}$ & $9,9(1,8) \mathrm{b}$ & $51,6(7,3) \mathrm{b}$ \\
PC & $14,8(1,5) \mathrm{b}$ & $60,7(3,8) \mathrm{ab}$ & $8,2(1,8) \mathrm{c}$ & $52,8(9,2) \mathrm{b}$ \\
MN & $14,6(1,3)$ & $56,3(3,7)$ & $9,6(2,7)$ & $50,8(9,4)$ \\
\hline
\end{tabular}

En que: Letras distintas en cada columna indican diferencias estadísticas entre tratamientos a un nivel de $\mathrm{P}<0,05$ mediante test de Tukey. Los valores entre paréntesis indican el desvío estándar. PI: preparación intensiva, ER: extracción de residuos, PC: conservación de residuos, MN: monte nativo. 
halló en PI. Además, en este tratamiento se advierte una caída importante $(\mathrm{P}<0,05)$ en la concentración de $\mathrm{CAF}$ en subsuperficie y las más bajas proporciones $\mathrm{AF} / \mathrm{CO} \quad(\mathrm{P}<0,05)$ en ambas profundidades de muestreo. Esto sería una consecuencia de dos factores. Por un lado, tal como lo establecen Fernández et al. (1997) y Gonzalez-Pérez et al. (2004) las quemas pueden provocar una evolución de las sustancias húmicas a un mayor grado de maduración, posiblemente a ácido humicos y/o huminas. Por otro lado, existiría el efecto adicional del laboreo generando la disminución de CAF por la ruptura de los agregados y la exposición del CO que estaba protegido (MIGLIERINA y ROSSEL, 1995; MARTIN et al., 1998).

Aunque no pueda someterse a una afirmación estadística podemos observar que los valores medios de CAF en MN superan a los hallados en las áreas con bosques cultivados luego de aplicar prácticas intensivas (PI) al inicio del primer y segundo ciclo forestal, similar a lo sucedido con el CL.

Con respecto a la fracción estable; el $\mathrm{CAH}$ representa entre el 30\% y el 37\% del CO del suelo entero siendo su correlación relativamente importante sólo en la profundidad $0-5 \mathrm{~cm}(\mathrm{r}=$ $0,59 ; \mathrm{P}<0,05)$. El CAH y la proporción $\mathrm{CAH} / \mathrm{CO}$ (Tabla 2) fue mayor $(\mathrm{P}<0,05)$ en ER para las dos profundidades, indicando una mayor estabilización de la MO. Las proporciones de $\mathrm{CAH}+\mathrm{CAF} /$ $\mathrm{CO}$ fueron significativamente más bajas en PI e indicarían que los compuestos orgánicos de media y baja estabilidad evolucionaron hacia la fracción no hidrolizable (CHU) (NASCIMENTO et al., 1993). Este aumento en el grado de transformación de la MO también se observó a través del aumento de la relación $\mathrm{CAH} / \mathrm{CAF}$ la que tomó un valor de 3,8 en PI; 3,7 en ER y 3,1 en PC. De manera similar a lo observado en este trabajo, BAYER et al. (1999) destacan un aumento en el grado de humificación de la MO nativa de un suelo tropical con bajos aportes de residuos.

La pérdida de $\mathrm{CAH}$ en PC (Tabla 2), asociada a la caída de $\mathrm{CO}$ particularmente en la capa 0-5 cm (Tabla 1), se debería a la biodegradación de estructuras de $\mathrm{C}$ más complejas de los $\mathrm{AH}$ en estructuras orgánicas más simples como AF.

El fraccionamiento químico evidenció que la mayor parte de $\mathrm{CO}$ del suelo (entre el $50 \%$ y el $70 \%$ ) se encuentra asociado a estructuras orgánicas de alta estabilidad (CHU); de allí la correlación detectada entre el CO del suelo entero y el CHU ( $r=$ $0,76 ; \mathrm{P}<0,05)$.
A pesar de la estabilidad o recalcitrancia de los compuestos orgánicos que conforman las huminas, se observó que éstas fueron modificadas según el sistema de manejo de residuos aplicado. Tanto la concentración de CHU como su proporción fue mayor $(\mathrm{P}<0,05)$ en el tratamiento PI (Tabla 2$)$, verificando lo planteado por Almendros et al. (1984) y Fernandez et al. (1997). Ante una quema una cierta proporción de los compuestos presentes en el extracto húmico total pasan a la forma no extraíble (CHU); manifestándose un incremento relativo en las fracciones más resistentes como las huminas de insolubilización (AH o AF ligados a arcillas y óxidos de Fe y Al). Simultáneamente, las fracciones de carácter termolábil (CAF) experimentaron una tendencia opuesta. Es posible hipotetizar que el aumento del CHU en PI provenga de restos de carbón presentes en la muestra de suelo (ANDERSON et al., 1974; STEVENSON y ELLIOT, 1989), siendo más notable en el espesor de muestreo $5-15 \mathrm{~cm}(\approx 70$ $\%$ ) por la translocación o mezcla de las partículas.

\section{CONCLUSIONES}

A dos años y cinco meses de realizar diferentes tratamientos a los residuos de la cosecha del Pinus elliotti no se observó un aumento en la concentración de $\mathrm{CO}$ y de $\mathrm{CL}$ donde estos fueron conservados en superficie (PC). Los resultados indicarían que, existen otros factores que controlaron la dinámica de la descomposición de los residuos y el ingreso de $\mathrm{C}$ al suelo.

$\mathrm{La}$ concentración de $\mathrm{CO}$ y $\mathrm{CL}$ en el tratamiento tradicional de la región (PI) fue similar a ER y ambos superiores a PC. La quema y el laboreo (PI) produjo una pérdida de CAF acompañada por un aumento en CHU y en la relación CAH/CAF. Esto pone en evidencia que PI genera un cambio en la calidad de la MO debido a una mayor estabilización.

Se requieren nuevas evaluaciones para constatar las tendencias observadas, particularmente luego del cierre de la canopia, con la formación del nuevo mantillo forestal.

\section{AGRADECIMIENTOS}

Al Proyecto Forestal de Desarrollo (SAGPyA-BIRF) y al FOMEC por la parcial financiación de este estudio. Al Sr. Eduardo Vella por su colaboración en las determinaciones de Laboratorio. A la empresa Alto Paraná SA por permitir la instalación del experimento. 


\section{REFERÊNCIAS BIBLIOGRÁFICAS}

ALMENDROS, G.; GONZÁLEZ-VILA，F. J.; MARTÍN, F. Fire-induced transformation of soil organic matter from an oak forest: an experimental approach to the effects of fire on humic substances. Soil Science v. 149, n. 3, p.158-168, marzo 1990. ANDERSON, D. W.; PAUL E. A.; ARNAUD, R .J. Extraction and characterization of humus with reference to clay associated humus. Can. J. Soil Sci. v. 54, n. 3, p.317-323, Aug. 1974.

BAYER, C.; MIELNICZUK, J.; NETO, L. M. Alterações da matéria orgânica associada a frações minerais induzidas pelo manejo do solo no sul do Brasil. In: ENCONTRO BRASILEIRO SOBRE SUBSTÂNCIAS HÚMICAS, 3., 1999. Santa María. Anais... Sana Maria, 1999, p.125-134.

CABRERA, A. L. Regiones fitogeográficas Argentinas. Enciclopedia Argentina de Agricultura y Jardinería. 3. ed. Argentina. ACME 1976. 85 p.

CADE-MENUN, B. J. et al. Phosphorus form and related soil chemistry of Podzolic soil on northern Vancouver Island. II The effects of clear-cutting and burning. Can. J. For. Res. v. 30, n. 11, p1726-1741, 2000.

CARTER, M. C.; FOSTER, C. D. Prescribed burning and productivity in southern pine forest: a review. Forest Ecol. and Management, v. 91, n. 1-3, p.93-109, Apr. 2004.

CHEN, C. R.; XU, C. Z. Soil carbon and nitrogen pool and microbial properties in a 6-year-old slash pine plantation of subtropical Australia: impacts of harvest residue management. Forest Ecol. and Management.v. 206, n. 1-3, p. 237-247, Feb. 2005. CROMACK, K. et al. Soil carbon and nutrients in a coastal Oregon Douglas fir plantation with red alder. Soil Sci. Soc. Am. J. v. 63, n. 1, p.232-239, Jan./Feb. 1999.

DEVELOPMENT CORE TEAM R: A language and environment for statistical computing. $\mathrm{R}$ Foundation for Statistical Computing, Vienna, Austria. ISBN 3-900051-00-3, URL http://www.Rproject.org. 2007.

DUXBURY, J. M.; SMITH, M. S.; DORAN, J. W. Soil organic matter as a source and a sink of plant nutrients. Dynamics of soil organic matter in tropical ecosystems. Honolulu, 1989. 245 p.

FEARNSIDE, P. M.; BARBOSA, R. I.. Soil carbon changes from conversion of forest to pasture in Brazilian Amazonia. Forest Ecology and Management v. 108, n.1-2, p.147-166, Aug. 1998. FERNÁNDEZ,A.; CABANEIRO, A; CARBALLA,
S. T. Organic matter changes immediately after a wildfire in an Atlantic forest soil and comparison with laboratory soil heating. Soil Biol. Boichemistry, v. 29, n. 1, p. 1-11, Jan. 1997.

FERNÁNDEZ, R. et al. Técnicas de manejo de residuos de cosecha para el establecimiento forestal y su impacto sobre la condición química de los suelos rojos del noreste de argentina. En: Avances en Ingeniería Agrícola. Facultad Agronomía, Universidad de Buenos Aires. 2000, p. 243:248.

GALANTINI, J. A.; SUÑER. L. Las fracciones orgánicas del suelo: análisis en los suelos de la Argentina. Agriscientia v. 25, n. 1, p. 41-55, junio 2008.

GIUFFRÉ, L. et al. Efecto de diferentes técnicas de manejo de residuos de la cosecha forestal en algunas propiedades de un suelo Kandiudult en el Noreste Argentino. Agricultura Técnica, v. 62, n. 1, p.143-149, enero 2002.

GONĢALVES, J. L. et al. Silvicultural effects on the productivity and wood quality of eucalypt plantations Forest Ecology and Management v. 193, n. 1-2, p. 45-61, mayo 2004.

GONZALEZ-PEREZ ET AL. The effect of fire on soil organic matter - a review. Environment International v. 30, n. 6, p. 855-870, Aug. 2004 GREGORICH, E.G.; JANZEN, H.H. Storage of soil carbon in the light fraction and macroorganic matter. Structure and organic matter storage in agricultural soils. Boca Raton: CRC Press, 1996. 167-192.

INTA. Atlas de suelo de la República Argentina. Provincia de Misiones, Secretaría de Agricultura, Ganadería y Pesca. Argentina. 1990, p.111-154.

IPCC. Good Practice Guidance for Land Use, LandUse Change, and Forestry Intergovernmental Panel of Climate Change. 2000. Disponible en: < (http:// www.ipcc.ch/publications_and_data/publications and_data_reports.htm) $>$ Acceso en diciembre 2009 . JURGENSEN, M. F. et al. Impacts of timber harvesting on soil organic matter, nitrogen, productivity, and health of Inland Northwest forests. Forest Sci. v. 43, n. 2, p 234-251, May 1997.

KÖGEL-KNABNER, I. et al. Advances in soil organic matter research: the impact on agriculture and the environment. Fate of plant components during biodegradation and humification in forest soils: Evidence from structural characterization of individual biomacromolecules. Special publication $\mathrm{N}^{\circ}$ 90. The Royal Society of Chemistry, Cambridge. 1991. 61-69.

KOPPEN, W.; GEIGER GRAZ UND, R. Munich. 
Band I, Teil C. Berlin. 1936.

KÖRSCHENS, M.; WEIGEL, A.; SCHULZ, E. Turnover of soil organic matter and long term balances- tools for evaluating sustainable productivity of soil. Z Pflanzenernahr Bodenk, v. 161, p. 409-424, 1998.

KUZYAKOV, Y.; FRIEDL, J. K.; STAHR K. Review of mechanisms and quantification of priming effects. Soil Biol. and Biochemistry, v. 32, n. 11, p. 1485-1498, Oct. 2000.

MARTIN, D. et al. Characteristics of humic substances in cultivated and natural forest soils of Sikkim. Geoderma, v. 84, n. 4, p. 345-362, July 1998.

MATHERS, N. J. et al. How does residue management impact soil organic matter composition and quality under Eucalyptus globules plantations in southwestern Australia?. Forest Ecol. and Management, v. 179, n. 1-3, p. 253-267, July 2003. MCKEE, W. H. Changes in soil fertility following prescribed burning on coastal plain pine sites. USDA Forest Service Research Paper 234, 1982, 23 p. MENDHAM, D. S. et al. Residue management effects on soil carbon and nutrient content and growth second rotation eucalypts. Forest Ecol. and Management, v. 181, n. 3, p. 357-372, Aug. 2003. MIGLIERINA, A.M.; ROSELL, R.A. Humus quantity and quality of an entic Haplustoll under different soil-crop management systems. Commun. Soil Sci. Plant Anan. v. 26, n. 19, p 3343-3355, Nov. 1995.

NAMBIAR, S. E. K. Sustained productivity of plantations: Science and practice. In: SIMPÓSIO IUFRO: MANEJO NUTRITIVO DE PLANTACIONES FORESTALES. Valdivia, 1995, p.388-393.

NASCIMENTO, V. M.; ALMENDROS, G.; FERNANDES, F. E.. Evolution patterns of the soil organic matter in some agricultural systems in the Brazilian "Cerrado" region. Eur. J. Soil Biol. v. 29, n. 3-4, p.177-182, 1993.

NEARY, D. G. et al. Fire effects on belowground sustainability: a review and synthesis. Forest Ecol and Management, v. 122, n. 1-2, p. 51-71, Sept. 1999.

NZILA, J. D. et al. The effects of slash management on nutrient cycling and tree growth in Eucalyptus plantation in the Congo. Forest Ecol. and
Management, v. 171, n. 1-2, p. 209-221, Nov. 2002. PICCOLO, G. A. Transformaciones de la materia orgánica de un suelo rojo (Kandihumult), durante el período de degradación y recuperación de la fertilidad. 1996. 117 p. Tesis (Maestria - Ciencias del Suelo)-Universidad de Buenos Aires. Buenos Aires, 1996.

POWERS R. F.et al. The North American long-term soil productivity experiment: finding from the first decade of research. Forest Ecol. and Management, v. 220, n. 1-3, p. 31-50, Dec. 2005.

RAISON, R. J. Modification of the soil environment by vegetation fires, with particular reference to nitrogen transformations: a review. Plant and Soil, v. 51, n. 1, p. 73-108, Feb. 1979.

RICHTER, M. et al. Densimetric fractionation of soil organomineral complexes. Journal of Soil Science v. 26, p. 112-123, 1975.

RICHTER, M.; MIZUNO, I.; SVARTZ, H. Un método rápido y preciso para la determinación de ácidos húmicos, ácidos fúlvicos y huminas en suelos. Revista de investigaciones agropecuarias. INTA, v. 4, n.2, p 25-36, 1978-1979

SHINDO, H.; URABE, M. Changes in the humus composition of volcanic ash soils by heating at various temperatures. Soil. Sci. Plant Nutrition v. 39, n. 1, p. 189-192, 1993.

SIX, J., R. T. et al. Stabilization mechanisms of soil organic matter: Implications for C-saturation of soils Plant and Soil. v. 241, n. 2, p. 155-176, Apr. 2002.

STEVENSON, F. J. Cycles of soil. Carbon, nitrogen, phosphorus, sulfur, micronutrients. 1986. 280 p.

STEVENSON, F. J.; ELLIOTT. E. Methodologies for assessing the quantity and quality of soil organic matter: Dynamics of soil organic matter in tropical ecosystems. Honolulu, University of Hawaii. 1989, 245 p.

USSIRI, D. A. N.; JOHNSON, C. H. Organic matter composition and dynamics in a northern hardwood forest ecosystem 15 years after clear-cutting. Forest Ecol. and Management.v. 240, n. 1-3, p 131-142, Mar. 2007.

WALKLEY, A.; BLACK, I. A. An examination of the Degtjareff method for determining soil organic matter and a proposed modification of the chromic acid titration method. Soil Sci. v. 37, p. 29-38, 1934. 